\title{
Community Health Management and Nursing Strategies for Elderly Hypertension
}

\begin{abstract}
Qize Zhong1*, Wanling Chen ${ }^{2}$
${ }^{1}$ Qianlinshan Habitat Community Health Service Center, People's Hospital of Longgang District, Shenzhen 517118, Guangdong Province, China

${ }^{2}$ Cancer Hospital Chinese Academy of Medical Sciences, Shenzhen Center, Shenzhen 517118, Guangdong Province, China

*Corresponding author: Qize Zhong, 517582780@qq.com

Abstract: Objective: To explore the therapeutic effects of community health management and nursing strategies for elderly hypertensive patients. Methods: A total of 64 elderly hypertensive patients who were treated in our hospital from March 2020 to March 2021 were selected. The control group took conventional care and guidance. The research group carried out community health management and nursing strategy guidance on the basis of the control group. Then compare the blood pressure levels of the two groups of patients before and after nursing and the patients' satisfaction with nursing. Results: Through comparison, it can be seen that the diastolic and systolic blood pressure levels of the study group and the control group are not significantly different before nursing. After nursing, the diastolic blood pressure of the patients in the study group was $81.22 .1 \mathrm{mmHg}$ and the systolic blood pressure was $126.58 .7 \mathrm{mmHg}$. The diastolic blood pressure of the control group was $90.55 .4 \mathrm{mmHg}$ and the systolic blood pressure was $136.412 .9 \mathrm{mmHg}$. There are obvious differences in the comparison of the two sets of data. By comparing the two groups of patients' satisfactions with nursing care, it can be seen that among the 32 patients in the study group: 31 were very satisfied and basically satisfied, with a satisfaction rate of $96.87 \%$. Among the 32 patients in the control group, 28 were very satisfied and basically satisfied, with a satisfaction rate of $87.5 \%$. The data of the two groups of patients are clearly comparable. Conclusion: Through community health management and nursing strategies, the satisfaction and treatment effect of elderly hypertensive patients can be improved, thereby contributing to the recovery of patients.
\end{abstract}

Keywords: Elderly; Hypertension; Community health management; Nursing strategy

Publication date: November 2021; Online publication: November 30, 2021

\section{Introduction}

Chronic diseases are diseases that have a slow onset and a long duration. This form of disease is more common among the elderly, owing to the patient's decreased physical function and resistance, which increases the risk of disease. At this time, hypertension is the most common clinical chronic condition. It basically refers to a condition in which the pressure value of the blood vessel wall is consistently greater than the normal value due to the patient's blood flowing in the blood vessel ${ }^{[1]}$. Patients with severe hypertension will develop coronary heart disease, stroke, and other disorders due to the disease's gradual start. Patients' health and lives will be jeopardised as a result of this. According to the findings of a Chinese epidemiological investigation, the prevalence of adult hypertension in China was as high as $27.9 \%$ from 2012 to 2015, equating to one hypertensive patient in every three individuals with enzymes. Currently, drug management is the primary method of treatment for this condition. As a high-risk group of diseases such as hypertension, it is of great significance for the elderly to adopt appropriate and effective management methods and nursing strategies to provide patients with targeted disease guidance ${ }^{[2-3]}$. Due to the obvious 
chronic nature of this condition, the community, as the primary group unit for patient treatment, plays a critical role in patient care. As a result, the therapeutic benefits of community health management and nursing interventions for elderly hypertension patients are the subject of this research. The present research has come to an end, and the following reports on the relevant content are now being prepared.

\section{Materials and methods}

\subsection{General information}

A total of 64 elderly hypertensive patients who were treated in our hospital from March 2020 to 2021 were selected. The diagnosis of the patient's condition met the clinical diagnostic criteria for hypertension, and patients with other major diseases were excluded. Following the voluntary principle of patients and their families to participate in this study, 64 patients were randomly divided into two groups: the study group had 32 patients, 19 males, and 13 females; the average age of the patients was 64.35.3years, and the course of the patients' illnesses. In 2.3-5.8 years, the average course of disease was 3.10.6 years. There were 32 patients in the control group, including 17 males and 15 females. The average age of the patients was 65.84.3 years. The patient's course of disease was 3.1-6.4 years, with an average course of 3.60.8 years. After statistically processing the general data of the two groups of patients, it can be found that there is no significant difference in the data and will not affect the study.

\subsection{Research methods}

The control group adopted conventional methods for nursing and guidance, including patient medication, dietary principles, and regular blood pressure measurement and condition review. The research group carried out community health management and nursing strategy guidance on the basis of the control group. The first is to establish medical records for patients in the community, and distinguish them based on the clinical data of the patients and the level of blood pressure. At the same time, organize patients in the community to participate in health promotion, seminars, etc., to popularize the pathological knowledge of the disease related to hypertension to patients. Enhance patients' understanding of the disease and compliance in the process of treatment and medication ${ }^{[4]}$. Due to the age of the patient, forgetting may occur. Therefore, pay attention to guiding the patients' medication, and provide detailed notifications of the time, method, and dosage of the medication. If necessary, write a note and give it to the patient, and emphasize to the patient that taking the medicine requires strict compliance with the doctor's advice. Avoid unauthorised adjustment of the dosage which may cause adverse reactions and affect your health. At the same time, the patient's diet and psychological aspects are guided to avoid the patient's adverse psychological condition due to the disease, which affects the rehabilitation and treatment effect. Secondly, in the process of community health management and nursing, it is necessary to establish a sufficient emergency plan. The risk assessment of complications that are likely to occur in older patients with higher blood pressure levels who have previous medical history is carried out. The factors are evaluated so that attention can be paid in the process of health work management ${ }^{[5]}$. At the same time, on the basis of distinguishing medical records, different targeted nursing methods are implemented for patients whose blood pressure meets and does not meet standards. For patients whose blood pressure meets the standard, a telephone or face-to-face interview is conducted every 4 weeks to ask about the patient's blood pressure, compliance with the doctor's advice, and physical condition. For patients whose blood pressure is not up to standard, a telephone or face-to-face interview is conducted every 2 weeks to communicate the patient's blood pressure control status. We provide targeted answers to some questions and psychological problems of patients, enhance patients' confidence in treatment, and improve patients' compliance with treatment. For severely ill patients, a face-to-face interview is conducted every $1 \mathrm{week}$, and the patients are asked about changes in their condition and their compliance with medications. In addition, we provide guidance 
for patients' family members to care for their condition, and improve the emergency response ability of patients in the event of an illness ${ }^{[6]}$. Finally, we regularly hold publicity conferences, exchange meetings and other activities to organize patients with better blood pressure control to share experience, organize exchanges, and enhance patients' confidence in recovery.

\subsection{Observation indicators}

Compare the blood pressure levels of the two groups of patients before and after nursing and the patients' satisfaction with nursing.

\subsection{Statistical processing}

The data analysis of this study used SPSS19.0 statistical software. The research data were processed and analyzed statistically, and the measurement data used the mean \pm standard deviation $( \pm \mathrm{s})$. The measurement data is expressed by the rate (\%), using the $\mathrm{X}^{2}$ test, and comparing the data before and after nursing in the same group using the paired t test. When $\mathrm{P}<0.05$, it is considered to be statistically significant, when $\mathrm{P}>0.05$, it is not statistically significant ${ }^{[7]}$.

\section{Results}

By comparison, it can be seen in Table 1. that the diastolic and systolic blood pressure levels of the study group and the control group are not significantly different before care. After nursing, the diastolic blood pressure of the patients in the study group was 81.22.1 $\mathrm{mmHg}$ and the systolic blood pressure was 126.58.7 $\mathrm{mmHg}$. The diastolic blood pressure of the control group was $90.55 .4 \mathrm{mmHg}$ and the systolic blood pressure was $136.412 .9 \mathrm{mmHg}$. There are obvious differences in the comparison of the two sets of data.

Table 1. Comparison of blood pressure levels before and after care of the two groups of patients $(\mathrm{mmHg})$

\begin{tabular}{lccccc}
\hline Groups & \multirow{2}{*}{$\begin{array}{c}\text { Number of } \\
\text { cases }\end{array}$} & \multicolumn{2}{c}{ Diastolic blood pressure } & \multicolumn{2}{c}{ Systolic blood pressure } \\
\cline { 3 - 5 } & 32 & Before caring & After caring & Before caring & After caring \\
\hline Research group & 32 & 97.43 .6 & 81.22 .1 & 154.35 .4 & 126.58 .7 \\
Control group & 32 & 97.94 .2 & 90.55 .4 & 153.54 .8 & 136.412 .9 \\
T value & & 0.983 & 6.486 & 0.526 & 7.424 \\
P value & & 0.653 & 0.002 & 0.784 & 0.000 \\
\hline
\end{tabular}

By comparing the two groups of patients' satisfactions with nursing care, it can be seen in Table 2 that among the 32 patients in the study group, 31 were very satisfied and basically satisfied, with a satisfaction rate of $96.87 \%$. Among the 32 patients in the control group, 28 were very satisfied and basically satisfied, with a satisfaction rate of $87.5 \%$. The data of the two groups of patients are clearly comparable.

Table 2. Comparison of patients' satisfaction with nursing care between the two groups

\begin{tabular}{lccccc}
\hline Groups & $\begin{array}{c}\text { Number of } \\
\text { cases }\end{array}$ & $\begin{array}{c}\text { Basically } \\
\text { satisfied }\end{array}$ & $\begin{array}{c}\text { Basically } \\
\text { satisfied }\end{array}$ & Dissatisfied & Satisfaction rate (\%) \\
\hline Research group & 32 & 19 & 12 & 1 & $96.87 \%$ \\
Control group & 32 & 15 & 13 & 4 & $87.5 \%$ \\
$X^{2}$ value & & & & & 6.492 \\
P value & & & & & 0.000 \\
\hline
\end{tabular}




\section{Discussion}

In this study, we discussed the therapeutic effects of community health management and nursing strategies for elderly hypertensive patients. We found that in terms of both blood pressure indicators and patient satisfaction indicators, the patients who implemented community health management were significantly better than those in the control group. From this, we can see that community health management is a continuation of hospital health management and treatment. It can improve the satisfaction and treatment effect of elderly hypertensive patients through community health management and nursing strategies, thereby contributing to the rehabilitation of patients ${ }^{[8]}$.

In summary, improving the level of community health management and nursing measures can improve the quality of treatment for patients.

\section{Disclosure statement}

The authors declare no conflict of interest.

\section{References}

[1] Dawa Z, 2019, The Application Effect of Health Education and Chronic Disease Management and Nursing in Community Elderly Hypertension. Health Vision, 000(014): 264-265.

[2] $\mathrm{Xu} \mathrm{G}, 2020$, Analysis of Community Health Management and Nursing Countermeasures for Hypertension and Diabetes in the Elderly. Health for All, 523(14): 369-370.

[3] Jiang M, Feng S, Liao J, 2017, Analysis of the Nursing Effect of the Implementation of Traditional Chinese Medicine Health Management for Elderly Hypertensive Patients in the Community. Modern Diagnosis and Treatment, 28(010): 1938-1940.

[4] Wu L, et al., 2017, The Effect of Community Health Management and Nursing Intervention on Hypertension and Diabetes in the Elderly. Chinese Community Physician, 15(33): 145-146.

[5] Liu X, 2019, The Effect of Community Health Management on Blood Pressure Control, Medication Compliance and Quality of Life in Elderly Hypertensive Patients. Dialysis and Artificial Organs, 30(3): 15-17.

[6] Zhao M, 2019, The Application Value of Community Health Management in the Control of Hypertension in Elderly Patients. Health Care Wenhui, 000(004): 212-213.

[7] Shi M, 2020, Study on Community Health Management and Nursing Strategies of Hypertension and Diabetes in the Elderly. Electronic Journal of Integrated Traditional Chinese and Western Medicine Cardiovascular Diseases, (32).

[8] Guo C, Sheng F, He X, 2020, Community Health Management and Nursing Strategies for Hypertension and Diabetes in the Elderly. China Health \& Nutrition, 030(011): 155-156. 